\section{Form and source of tocopherol affects Vitamin E status in Thoroughbred horses}

\author{
Joe D. Pagan, Ed Kane and Delia Nash \\ Kentucky Equine Research Inc., Versailles, Kentucky USA
}

\begin{abstract}
Introduction
Vitamin E functions as a biological antioxidant, preventing the oxidation of unsaturated lipid materials within cellular and subcellular membranes by neutralizing production of free radicals. It is an important nutrient for all horses but is especially important for exercise, reproduction, and for horses at risk of certain neurological diseases. Mayhew et al. (1987) found that supplementing mares with $1500 \mathrm{IU}$ of vitamin $\mathrm{E}$ per day decreased the incidence of equine degenerative myeloencephalopathy (EDM) in foals born to these mares from $40 \%$ to $10 \%$. Blythe and Craig (1993) found that young foals showing signs of incoordination and ataxia appeared normal by two years of age when supplemented with $6000 \mathrm{IU}$ of vita-
\end{abstract} $\min \mathrm{E}$.

Vitamin E can be obtained from natural or synthetic sources, but the chemical structure of each is different. Natural vitamin $E$ is composed of one isomer ( $d$ - $\alpha$-tocopherol [RRR $\alpha$-tocopherol]), while synthetic vitamin $E$ is a mixture of eight isomers (dl- $\alpha$-tocopherol [all-rac- $\alpha$-tocopherol]), of which only one or $12.5 \%$ is identical to the natural isomer. Vitamin $E$ is typically added to equine feeds in an esterifed form ( $\alpha$-tocopherol acetate) to prolong shelf life. In order for vitamin E acetates to be utilized in the body, two steps are necessary: the ester has to be removed and the alpha-tocopherol has to be made water soluble by the action of bile salts in a process known as micellization. Each of these steps may limit vitamin E absorption in the horse.

\section{Materials and Methods}

Three studies were conducted to assess the effects of different forms of vitamin $E$ on vitamin $E$ status. Vitamin $E$ treatments used in these studies included synthetic vitamin $E(d)-\alpha$ tocopherol acetate) (SYN), natural-source vitamin $E(d-\alpha$ tocopherol acetate) (NAT) ${ }^{1}$, and micellized natural vitamin $E$ $(d \text { - } \alpha \text {-tocopherol) (WS })^{2}$. In each of these studies, vitamin $E$ status was assessed by measuring plasma levels of $\alpha$-tocopherol by reversed-phase HPLC (Sowell et al. 1994).

In study 1, SYN and NAT were assessed in exercised horses (four Arabian and four Thoroughbred geldings). Each of these horses had been in a regular training program for several months prior to commencement of the study and were considered physically fit. The study consisted of four periods: period 1, 4-week depletion; period 2, 6-week repletion; period
3, 4-week depletion; and period 4, 6-week repletion. The study utilized a switchback design with a supplementation level of $1000 \mathrm{mg} /$ day. Horses were maintained on a diet of grass hay and unfortified sweet feed in a 60:40 ratio. A vitamin and mineral premix that contained no vitamin $E$ was also fed. Horses wore muzzles during turnout to prevent grazing. At the end of each period, horses underwent a standardized exercise test (SET) on the treadmill. Blood samples for measurement of vitamin $\mathrm{E}$ content were taken immediately before exercise and at $5 \mathrm{~min}, 30 \mathrm{~min}, 4 \mathrm{hr}, 24 \mathrm{hr}$, and $48 \mathrm{hr}$ post exercise.

In study 2, nine mature unexercised Thoroughbred geldings were divided into three groups and supplemented with SYN, NAT, or WS. Horses were maintained on a diet of unfortified sweet feed and grass hay, and wore muzzles during turnout to prevent grazing. Baseline blood samples were taken following 14 days of no supplementation. Horses were then supplemented with $500 \mathrm{IU}$ of vitamin $\mathrm{E}$ from the assigned treatment source for 14 days. The dose rate of vitamin $E$ was doubled at 14-day increments up to $8000 \mathrm{IU}$. Plasma tocopherol levels were measured at the end of each 14-day period.

In study 3, nine unexercised Thoroughbred geldings were supplemented for 56 days with 5000 IU of vitamin E as SYN, NAT, or WS. Horses were maintained on the same basal diet as study 2 and wore muzzles during turnout to prevent grazing. Horses had not received any form of vitamin E supplementation for 6 weeks prior to commencement of the study. Baseline blood samples were taken prior to supplementation and at weekly intervals during the supplementation period for measurement of plasma tocopherol levels.

\section{Results}

Study 1

Plasma tocopherol levels were significantly higher $(P<0.05)$ in the SYN and NAT groups compared to depletion levels except in the pre-exercise blood sample where the levels in the SYN group were not significantly higher than depletion levels. Horses supplemented with NAT exhibited higher plasma tocopherol levels before and after exercise compared to those supplemented with SYN $(P<0.05)$ (Figure 1).

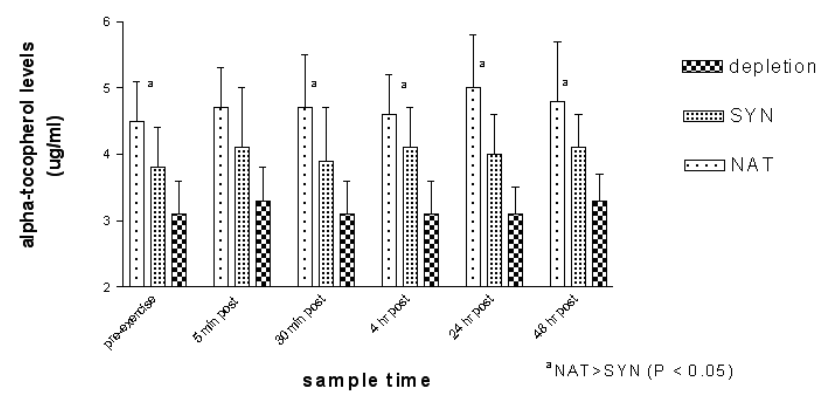

Fig 1 Plasma vitamin E levels pre- and post-exercise.

\section{Study 2}

Plasma tocopherol levels were significantly elevated from baseline in the WS and NAT groups at $1000 \mathrm{IU}$ through 8000 
$\mathrm{IU}$ and $4000 \mathrm{IU}$ through $8000 \mathrm{IU}(\mathrm{P}<0.001)$, respectively. No significant difference was recorded in the SYN group as dose rate was increased. Significant differences between groups $(\mathrm{P}<0.001)$ were recorded at $1000 \mathrm{IU}$ (WS $>$ SYN), $2000 \mathrm{IU}$ (WS > NAT > SYN), $4000 \mathrm{IU}(\mathrm{WS}>$ NAT > SYN), and $8000 \mathrm{IU}$ (WS $>$ NAT $>$ SYN) (Figure 2).

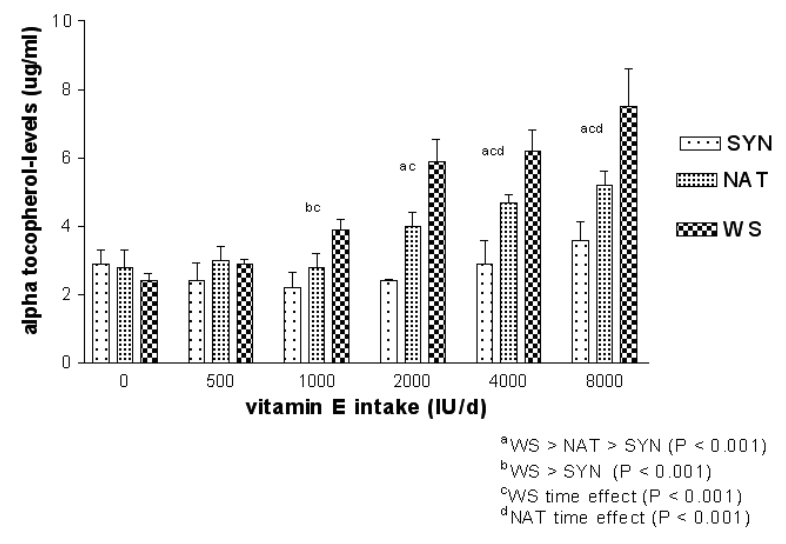

Fig 2 Plasma vitamin E following 14-day intervals of increasing levels of vitamin E supplementation.

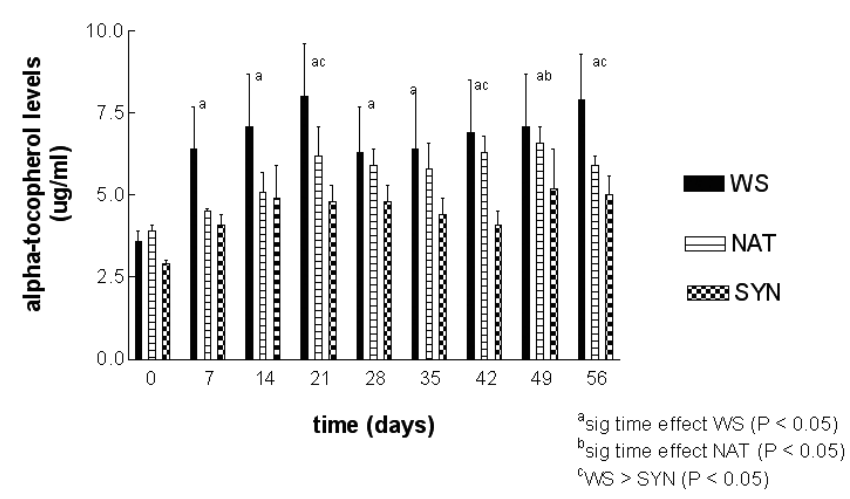

Fig 3 Plasma vitamin E concentrations with 5000 IU of vitamin E supplementation.

Study 3

No significant differences were evident in the SYN group over time. A significant time effect was recorded in the WS group on days 7 through 56 and in the NAT group on day 49 $(P<0.05)$. Plasma tocopherol levels were raised significantly in the WS group compared to the SYN group $(\mathrm{P}<0.05)$ on days 21,42 , and 56 (Figure 3).

\section{Discussion and Conclusion}

Results from these studies indicate that the source of vitamin E significantly affects vitamin E status in exercised and unexercised horses. Synthetic vitamin E (dl- $\alpha$-tocopherol acetate) was less effective at elevating plasma tocopherol levels than natural source vitamin $E$, and a micellized form of vitamin $E$ was superior at elevating plasma tocopherol during shortterm administration.

\section{Manufacturer addresses}

1 KER Equine Ester, Kentucky Equine Research, Inc., Versailles, KY

2 Elevate WS, Kentucky Performance Products, Versailles, KY

\section{References}

Blythe L. L. and Craig A. M. (1993): Equine degenerative myeloencephalopathy. Part 1. Clinical signs and pathogenesis. Equine Compedium 14, 1215

Mayhew I. G., Brown C. M., Stowe H. D., Trapp A. L., Derksen F. J. and Clement S. F. (1987): Equine degenerative myeloencephalopathy: A vitamin E deficiency that may be familial. J. Vet. Intern. Med. 1, 45

Sowell A. L., Huff D. L., Yeager P. R., Caudill S. P. and Gunter E. W. (1994): Retinol, alpha-tocopherol, lutein/zeaxanthin, beta-cryptoxanthin, lycopene, alpha-carotene, trans beta-carotene, and four retinyl esters in serum determined simultaneously by reversed-phase HPLC with multiwavelength detection. Clin. Chem. 40, 411416 